\title{
Lichenoide und pseudolymphomatöse Reaktion auf rotes Permanent-Tattoo „Schweizerkreuz“
}

\author{
Lichenoid Red Permanent Tattoo Hypersensitivity to „Flag of Switzerland“
}

Autoren

Institute
T. Plaza', W. Kempf ${ }^{2}$

Hautarztpraxis, Uster/Schweiz

kempf und pfaltz. histologische diagnostik, Zürich/Schweiz

\section{Bibliografie}

DOI http://dx.doi.org/

10.1055/s-0030-1255684

Online-Publikation: 17. 8. 2010

Akt Dermatol 2010; 36:

374-376 @ Georg Thieme

Verlag KG Stuttgart · New York ISSN 0340-2541

Korrespondenzadresse

Dr. med. Tobias Plaza

Hautarztpraxis

Brunnenstraße 9

8610 Uster

Schweiz

info@hautarzt-uster.ch

\section{Zusammenfassung \\ $\nabla$}

Wir berichten von einem 25-jährigen Patienten, bei dem es drei Wochen nach Tätowierung eines roten "Schweizerkreuzes“ zu einer scharf begrenzten, stark juckenden und teilweise erosiven Reaktion im Bereich des Tattoos kam. Die Histologie zeigte eine lichenoide und pseudolymphoma-

\section{Fallbericht \\ $\nabla$}

\section{Anamnese}

Bei Erstvorstellung im Juli 2009 berichtet der 25jährige Bankangestellte, dass er und drei Freunde sich an Silvester 2008 nach Käsefondue, mehreren Flaschen Bier und einer Flasche Kirschwasser gegenseitig im Keller des einladenden Freundes tätowiert hätten. Einen entsprechenden Profi-Permanent-Tattoo-Kasten habe einer der Freunde zuvor im Internet aus Asien bestellt und mitgebracht.

Bereits 3 Wochen nach Tätowierung des roten „Schweizerkreuzes“ sei es zu Juckreiz und Schwellung gekommen ( $\bullet$ Abb. 1). Die Symptome hätten seither stetig zugenommen, sodass er sich nun im Juli 2009 hier vorstelle.

Auf den schwarzen Rahmen sowie das schwarze Datum des Rütlischwures des gleichen Tattoos sei es zu keiner Reaktion gekommen. Ein außerdem seit 3 Jahren bestehendes schwarzes Permanent-Tattoo aus dem Tattoo-Studio habe nie Probleme bereitet. Auch sonst habe er abgesehen von einer leichten Akne als Teenager nie Hautprobleme gehabt, außerdem weder bekannte Allergien, Heuschnupfen noch Asthma bronchiale; keine regelmäßige Medikamenteneinnahme.

Unter Würdigung der Anamnese bestand der Verdacht, dass ein Inhaltsstoff des roten PermanentTattoos für die Beschwerden des Patienten verantwortlich sein könnte. Daher entschieden wir uns zur Epikutantestung der DKG-Standardreihe, töse Reaktion ohne granulomatöse Veränderungen. Die diagnostische Bestätigung einer Typ IVAllergie auf die verwendete Tattoo-Farbe erweist sich als schwierig, weil eine Epikutantestung auf wasserunlösliche Farbpigmente wahrscheinlich wenig sensitiv ist und eine Intrakutantestung das Risiko langwieriger Hautschäden mit sich bringt.

DKG-Friseurstoffe, P-Phenylendiamin, DKG-Dentalmetalle, DKG-Leder- und Textilfarben, DKGKonservierungsstoffe, Pigment Rot 170 sowie der mitgebrachten roten Tattoo-Farbe ( $\bullet$ Abb. 2). Außerdem führten wir eine Pricktestung auf Inhalativa sowie eine Punchbiopsie zur histologischen Untersuchung durch.

\section{Klinischer Befund bei Vorstellung}

Im Bereich des roten Tattoos am linken Unterschenkel eine lichenoide erythematöse Papelbildung mit starker Exkoriation ( $\bullet$ Abb. 1). Die restliche Haut ist komplett erscheinungsfrei, insbesondere keine Anzeichen für einen Lichen ruber der Haut oder Schleimhaut oder einen Lupus erythematodes.

\section{Allergologische Befunde \\ Epikutantestung}

Die Epikutantestung wurde nach Standardverfahren durchgeführt [1]. Die Ablesung erfolgte nach 48, 72, 96 Stunden sowie nach einer Woche.

Epikutantestung komplett negativ, inklusive der mitgebrachten Tattoo-Farbe.

\section{Pricktestung}

Die Pricktestung auf Inhalativa wurde nach Standardverfahren durchgeführt [2]. Die Ablesung erfolgte nach 20 und 40 Minuten.

Negativ: Katzenepithelien (Allergopharma), Hundeepithelien (Allergopharma), Pferdehaar (Allergopharma), Graspollen (Allergopharma), Roggen 

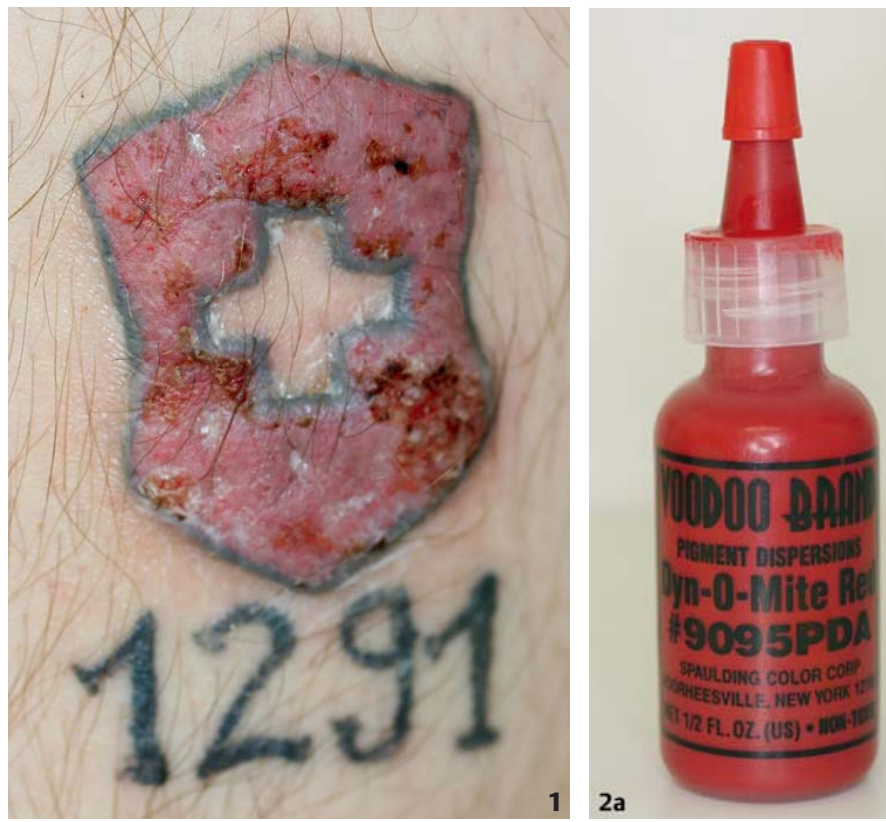

Abb. 1 Lichenoide Reaktion auf Permanent-Tattoo „Schweizerkreuz".

Abb. 2 a Verwendete Tattoo-Farbe „Woodo Brand“.

b Warnhinweise auf Farbverpackung ohne Angabe der Inhaltsstoffe.

(Allergopharma), frühblühende Baumpollen (Allergopharma), mittelblühende Baumpollen (Allergopharma), Ragweed (Allergopharma); Blumenmischung (Allergopharma), Kräutermischung (Allergopharma), Dermatophagiodes pteronyssinus (Allergopharma), Dermatophagoides farinae (Allergopharma), Latexmilch (Dalhausen). Dreifach-positive Histaminkontrolle, negative 0,9\% NaCl-Kontrolle.

\section{Histologie}

Epidermis mit Akanthose und fokaler pseudoepitheliomatöser Hyperplasie, fokaler Vakuolisierung im Bereich der Verbundzone mit Exozytose lymphozytärer Zellen insbesondere im Bereich der verlängerten Reteleistenspitzen. Fokale hyperparakeratotische Verhornung insbesondere im Bereich eines Follikelostiums. Herdförmige Ulzeration mit Auflagerung von Bakterien und neutrophilen Granulozyten. In sämtlichen Dermisschichten findet sich ein perivaskulär betontes und in den oberflächlichen Anteilen teils auch angedeutet bandförmiges dichtes lymphohistiozytäres Infiltrat. Beimengung vereinzelter Plasmazellen und vereinzelter eosinophiler Granulozyten. Keine Darstellung von exogenem Pigment. PAS-Färbung unauffällig. Die Befunde entsprechen einer lichenoiden und pseudolymphomatösen Reaktion, wobei die lichenoide Komponente angesichts der epithelialen Hyperplasie Züge eines hypertrophen Lichen ruber aufweist. Das typische Bild einer kontaktallergischen ekzematösen Reaktion liegt nicht vor ( $\bullet$ Abb. 3 a u. b).

\section{Diskussion}

Seit Beginn der Zivilisation sind Tätowierungen Teil der Menschheitsgeschichte. Es kann davon ausgegangen werden, dass wahrscheinlich jede Kulturgemeinschaft der Erde zu irgendeinem Zeitpunkt ihrer Entwicklung die Sitte des Tätowierens kannte und ausübte. Die ältesten Belege für das Vorkommen von Tätowierungen aus Europa stammen aus der Bronzezeit. Die 1991 entdeckte Mumie vom Hauslabjoch in der Nähe des Ötztales in Italien („Ötzi“) mit ihren 15 Hautbildern beweist, dass bereits vor mehr als 5000 Jahren in Europa Ornamente in die Haut gestochen wurden.
Bei unserem Fall handelt es sich um einen jungen Mann, der eine lichenoide Reaktion auf ein Permanent-Tattoo in Form eines Schweizerkreuzes entwickelt hat.

Das Schweizerkreuz wurde bereits von den alten Eidgenossen als Feldzeichen verwendet, die früheste dokumentierte Verwendung geht auf die Schlacht von Laupen im Jahr 1339 zurück. Ursprünglich waren die Arme des Kreuzes schmaler, ähnlich wie in den skandinavischen Flaggen. Offizielle Hoheitszeichen waren aber in der alten Eidgenossenschaft nur die Wappen der Kantone. 1815 wurde das Kreuz zum offiziellen Schweizer Wappen, es fand zunächst aber nur auf dem Schweizer Siegel Verwendung. Erst am 12. Dezember 1889, 41 Jahre nach der Gründung des Bundesstaates, legte die Bundesversammlung die heutige Form eines frei stehenden, aufrechten, weißen Kreuzes auf rotem Felde mit einem Seitenverhältnis der Arme von 7:6 fest.

Mit der zunehmenden Mode permanenter Körperkunst (Häufigkeit in Deutschland 8,5\% der Bevölkerung) [3] werden vermehrt auch allergische Reaktionen oder Irritationen beobachtet $[4,5]$. Roter Tattoo-Farbstoff ist die häufigste Ursache für unerwünschte Reaktionen auf Permanent-Tattos. Beschrieben wurden granulomatöse [6-8], ekzematöse [9], pseudolymphomatöse [10] und, wie im geschilderten Fall, lichenoide [7,11-16,21] Reaktionen. Lichenoide Reaktionen wurden meist auf Quecksilber $[7,12,14]$ und seltener auf Nickel [13] oder Azofarbstoffe [11,17,18] nachgewiesen. Dennoch gab es auch Fälle, bei denen weder Reaktionen auf Nickel oder Quecksilber noch auf die eigenen getesteten Farbstoffe im Epikutantest nachgewiesen werden konnten [1618].

Dass wir in unserem Fall keinen eindeutigen Sensibilisierungsnachweis erbringen konnten, schließt eine allergische Ursache nicht aus. Der histologische Befund einer lichenoiden und pseudolymphomatösen Reaktion korreliert mit der Hypothese einer Allergie und kann als Ausdruck einer Spättypreaktion gewertet werden. In anderen Fallberichten gab es ebenfalls negative Epikutantestreaktionen, bei dann allerdings positiver Intrakutantestung von Quecksilber-II-Amidochlorid oder dem Azofarbstoff Pigment Rot 170 [16-18]. Eine mögliche Erklärung dafür wäre, dass beispielsweise die schwer wasserlöslichen Azofarbstoffe kaum durch die Epidermis penetrieren. Ob es sich bei dem tätowierten roten Farbstoff aus Asien um einen Azofarbstoff wie Pig- 


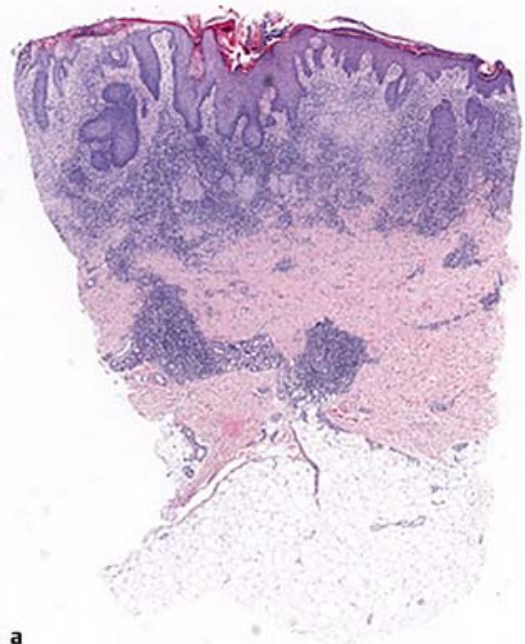

Abb. 3 Histologie mit lichenoider und pseudolymphomatöser Reaktion im Bereich des roten PermanentTattoos; (a) Übersicht, (b) Detailaufnahmen.

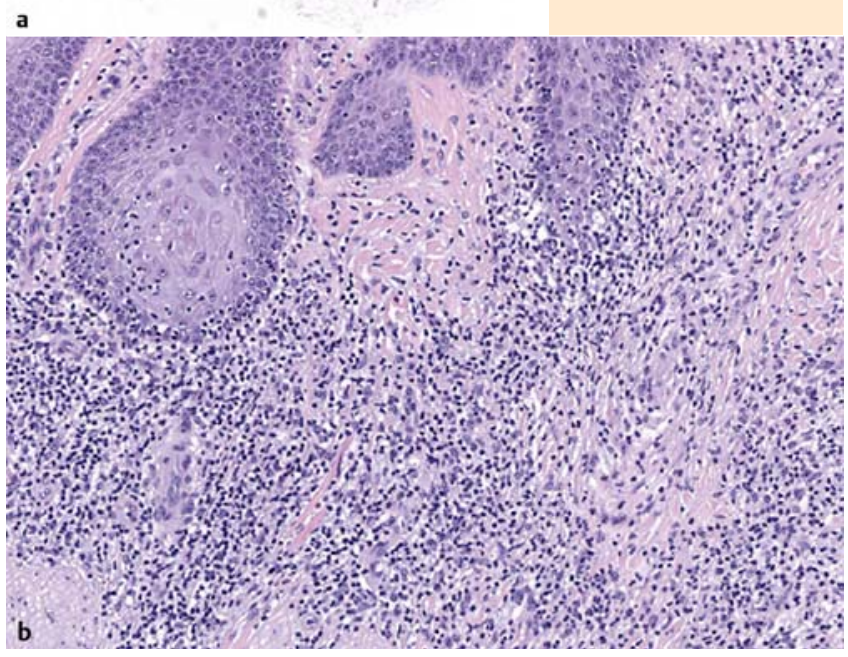

ment Rot 170 handelt, ließ sich nicht eruieren. Die vorgeschlagene Intrakutantestung mit Quecksilber, Pigment Rot 170 und dem eigenen Tattoo-Farbstoff lehnte der Patient aufgrund der möglicherweise langanhaltenden Folgeschäden an der Haut strikt ab. Die Therapie lichenoider Reaktionen auf Permanent-Tattoos erweist sich als äußerst schwierig. Ein konservativer Therapieversuch mit Dermovate-Creme ${ }^{\circledR}$ okklusiv brachte in unserem Fall nur kurzfristige Besserung mit raschem Rezidiv der Symptome bei Ausschleichen des topischen Steriods. Ein anschließend eingeleiteter Therapieversuch mit Protopic-Salbe ${ }^{\circledR}$ [20] führte zu keiner Besserung. Einzelne Fallberichte von erfolgreichen Lasertherapien mit dem Q-switched ND:YAG-Laser [21] oder dem Erbium:YAG-Laser [19] sind in der Literatur beschrieben, aufgrund der fraglichen Erfolgschancen, der möglichen Entstehung von karzinogenen Spaltprodukten [22] und der hohen Therapiekosten entschied sich unser Patient letztlich für die Exzision in toto mit anschließender Meshgraft-Deckung des Defekts am Unterschenkel.

Zusammenfassend sind Überempfindlichkeitsreaktionen im Verhältnis zu der Häufigkeit von Tattoos eher selten, gewinnen aber wegen der zunehmenden Anzahl an Tätowierten stetig an Bedeutung. Die klinische Verdachtsdiagnose einer allergischen Reaktion findet sich leicht, der diagnostische Nachweis und die Therapie erweisen sich jedoch als schwierig.

\section{Abstract}

\section{Lichenoid Red Permanent Tattoo Hypersensitivity to „Flag of Switzerland“s \\ $\nabla$}

We report on a 25-year-old male patient suffering three weeks after the application of a permanent tattoo from strongly infiltrated and slightly scaly eruptions restricted to the red-colored areas. Histology revealed lichenoid and pseudolymphomatous dermatitis without signs of granulomatous reaction. The verification of allergic sensitization in hypersensitivity reactions to tattoos by patch testing may be difficult due to the poor penetration into the skin of the applied color pigments. Intradermal testing may be more sensitive but bears the risk of long lasting skin reactions.

\section{Literatur}

1 Schnuch A, Aberer W, Agathos M et al. Leitlinien der Deutschen Dermatologischen Gesellschaft (DDG) zur Durchführung des Epikutantests mit Kontaktallergenen. Hautarzt 2001; 52: 864-866

2 Dreborg S. EAACI: skin tests for diagnosis of IgE-mediated allergy. Allergy 1989; 44 (Suppl 10): 31 - 37

3 Stirn A. Mein Körper gehört mir - Kulturgeschichte und Psychologie des Piercings und Tattoo. Therapeutische Umschau 2007; 64: 115119

4 Anderson R. Regarding Tattoos. Arch Dermatol 2001; 137: 210-212

5 Goldstein N. Tattoos today. Arch Dermatol 1985; 121: 604-605

6 Schmidt H, Christensen HE. Red poster paint tattoo granuloma. Arch Dermatol 1978; 114: 965-966

7 Sowden JM, Byrne JPH, Smith AG et al. Red Tattoo reactions. Xray microanalysis and patch-test studies. Br J Dermatol 1991; 124: 576-580

8 Verdich J. Granulomatous reaction in red tattoo. Acta Derm Venerol 1980; 61: 176-177

9 Pauluzzi P, Giordani M, Guarneri GF, Pascone M. Chronic eczematous reaction to red tattoo. J Eur Acad Dermatol Venereol 1998; 11: 187-188

10 Blumenthal G, Okun MR, Pontich JA. Pseudolymphomatous reaction to tattoos. J Am Acad Dermatol 1982; 6: 485- 488

11 Bendsoe N, Hansson C, Sterner O. Inflammatory reactions from organic pigments in red tattoos. Acta Derm Venereol 1991; 71: 70-73

12 Clarke J, Black MM. Lichenoid tattoo reactions. Br J Dermatol 1979; 100: $451-454$

13 Corazza M, Zampino MR, Montanari A et al. Lichenoid reaction from a permanent red tattoo: has nickel a possible aetiologic role? Contact Dermatitis 2002; 46: 114-115

14 Hindson C, Foulds I, Cotterill J. Laser therapy of lichenoid red tattoo reaction. Br J Dermatol 1995; 133: 665-666

15 Taaffe A, Knight AG, Marks R. Lichenoid tattoo hypersensitivity. Br Med J 1978; $1: 616-618$

16 Tresukosol P, Ophaswongse S, Kullavanijaya P. Cutaneous reaction to cosmetic lip tattooing. Contact Dermatitis 1997; 36: 176-177

17 Greve B, Chytry R, Raulin C. Contact dermatitis from red tattoo pigment (quinacridone) with secondary spread. Contact Dermatitis 2003; 49: $265-266$

18 Waldmann I, Vakilzadeh F. Delayed type allergic reaction to red azo dye in tattooing. Hautarzt 1997; 48: 666-670

19 De Argila D, Chaves A, Moreno JC. Erbium:Yag laser therapy of lichenoid red tattoo reaction. J Eur Acad Dermatol Venereol 2004; 18: 332 - 333

20 Campbell FA, Gupta G. Lichenoid tattoo reaction responding to topical tacrolimus ointment. Clin Exp Dermatol 2006; 31: 293-294

21 Antony FC, Harland CC. Red ink tattoo reactions: successful treatment with the Q-switched Nd:YAG laser. Br J Dermatol 2003; 149: 94 - 98

22 Bäumler W, Eibler ET, Hohenleutner U et al. Q-switch laser and tattoo pigments: first results of the chemical and photophysical analysis of 41 compounds. Lasers Surg Med 2000; 26: $13-21$

23 Amann U, Luger TA, Metze D. Lichenoid pseudolymphomatous tattooing reaction. Hautarzt 1997; 48: 410-413 